PROCEEDINGS OF THE

AMERICAN MATHEMATICAL SOCIETY

Volume 133, Number 10, Pages 2829-2834

S 0002-9939(05)07879-2

Article electronically published on March 24, 2005

\title{
ON THE ABSENCE OF UNIFORM DENOMINATORS IN HILBERT'S 17TH PROBLEM
}

\author{
BRUCE REZNICK
}

(Communicated by Michael Stillman)

\begin{abstract}
Hilbert showed that for most $(n, m)$ there exist positive semidefinite forms $p\left(x_{1}, \ldots, x_{n}\right)$ of degree $m$ which cannot be written as a sum of squares of forms. His 17th problem asked whether, in this case, there exists a form $h$ so that $h^{2} p$ is a sum of squares of forms; that is, $p$ is a sum of squares of rational functions with denominator $h$. We show that, for every such $(n, m)$ there does not exist a single form $h$ which serves in this way as a denominator for every positive semidefinite $p\left(x_{1}, \ldots, x_{n}\right)$ of degree $m$.
\end{abstract}

\section{INTRODUCTION}

Let $H_{d}\left(\mathbb{R}^{n}\right)$ denote the set of real homogeneous forms of degree $d$ in $n$ variables (" $n$-ary $d$-ics"). By identifying $p \in H_{d}\left(\mathbb{R}^{n}\right)$ with the $N=\left(\begin{array}{c}n+d-1 \\ n-1\end{array}\right)$-tuple of its coefficients, we see that $H_{d}\left(\mathbb{R}^{n}\right) \approx \mathbb{R}^{N}$. Suppose $m$ is an even integer. A form $p \in H_{m}\left(\mathbb{R}^{n}\right)$ is called positive semidefinite or psd if $p\left(x_{1}, \ldots, x_{n}\right) \geq 0$ for all $\left(x_{1}, \ldots, x_{n}\right) \in \mathbb{R}^{n}$. Following [1], we denote the set of psd forms in $H_{m}\left(\mathbb{R}^{n}\right)$ by $P_{n, m}$. Since $P_{n, m}$ is closed under addition and closed under multiplication by positive scalars, it is a convex cone. In fact, $P_{n, m}$ is a closed convex cone: if $p_{n} \rightarrow p$ coefficient-wise, and each $p_{n}$ is psd, then so is $p$. A psd form is called positive definite or $p d$ if $p\left(x_{1}, \ldots, x_{n}\right)=0$ implies $x_{j}=0$ for $1 \leq j \leq n$. The pd $n$-ary $m$-ics are the interior of the cone $P_{n, m}$.

A form $p \in H_{m}\left(\mathbb{R}^{n}\right)$ is called a sum of squares or sos if it can be written as a sum of squares of polynomials; that is, $p=\sum_{k} h_{k}^{2}$. It is easy to show in this case that each $h_{k} \in H_{m / 2}\left(\mathbb{R}^{n}\right)$. Again following [1], we denote the set of sos forms in $H_{m}\left(\mathbb{R}^{n}\right)$ by $\Sigma_{n, m}$. Clearly, $\Sigma_{n, m}$ is a convex cone; less obviously, it is a closed cone, a result proved in general by R. M. Robinson [22], although shown for $\Sigma_{3,6}$ by Hilbert [9].

In light of the inclusion $\Sigma_{n, m} \subseteq P_{n, m}$, let $\Delta_{n, m}=P_{n, m} \backslash \Sigma_{n, m}$. It was well known by the late 19 th century that $P_{n, m}=\Sigma_{n, m}$ when $m=2$ or $n=2$. In 1888, Hilbert proved [9] that $\Sigma_{3,4}=P_{3,4}$; more specifically, every $p \in P_{3,4}$ can be written as the sum of three squares of quadratic forms. (An elementary proof, with "five"

Received by the editors May 19, 2003 and, in revised form, May 24, 2004.

2000 Mathematics Subject Classification. Primary 11E10, 11E25, 11E76, 12D15, 14P99.

This material is based in part upon work of the author, supported by the USAF under DARPA/AFOSR MURI Award F49620-02-1-0325. Any opinions, findings, and conclusions or recommendations expressed in this publication are those of the author and do not necessarily reflect the views of these agencies.

(C)2005 American Mathematical Society Reverts to public domain 28 years from publication 
squares is in [2, pp. 16-17]; for modern expositions of Hilbert's proof, see [26] and [23.) Hilbert also proved in [9] that the preceding are the only cases for which $\Delta_{n, m}=\emptyset$. That is, if $n \geq 3$ and $m \geq 6$ or $n \geq 4$ and $m \geq 4$, then there exist psd $n$-ary $m$-ics that are not sos.

In 1893, Hilbert [10] generalized his three-square result for $P_{3,4}$ to ternary forms of higher degree. Suppose $p \in P_{3, m}$ with $m \geq 6$. Then there exist $p_{1} \in P_{3, m-4}$ and $h_{1 k} \in H_{m-2}\left(\mathbb{R}^{3}\right), 1 \leq k \leq 3$, so that

$$
p_{1} p=h_{11}^{2}+h_{12}^{2}+h_{13}^{2} .
$$

(Hilbert's proof seems to be non-constructive and lacks a modern exposition. In the very recent paper [11], de Klerk and Pasechnik discuss the implementation of an algorithm to find $p_{1}$ so that $p_{1} p$ is sos, though not necessarily as a sum of three squares. This paper uses Hilbert's result without giving an independent proof.)

If $m=6$ or 8 , then $p_{1}$ is a sum of three squares of forms, and hence (as Landau later noted [12]), the four-square identity implies that $p_{1}^{2} p=p_{1}\left(p_{1} p\right)$ is the sum of four squares of forms. If $m \geq 10$, then the argument can be applied to $p_{1}$ : there exists $p_{2} \in P_{3, m-8}$ with $p_{2} p_{1}=h_{21}^{2}+h_{22}^{2}+h_{23}^{2}$. Thus, if $m=10$ or 12 (so that $\left.P_{3, m-8}=\Sigma_{3, m-8}\right)$, then $\left(p_{1} p_{2}\right)^{2} p=p_{2}\left(p_{2} p_{1}\right)\left(p_{1} p\right)$ is the sum of four squares of forms. An easy induction shows that there exists $q \in H_{t}\left(\mathbb{R}^{3}\right)$ with $t=\left\lfloor\frac{(m-2)^{2}}{8}\right\rfloor$ so that $q^{2} p$ is the sum of four squares of forms.

Hilbert's 17th Problem asked whether this generalizes to $n>3$ variables; that is, if $p \in P_{n, m}$, must there exist some form $q$ so that $q^{2} p$ is sos? Artin proved that there must be, in a way that gives no information about $q$. Much more on the history of this subject can be found in the survey paper [20].

This discussion leads to two closely related questions. Suppose $p \in P_{n, m}$. Can we find a form $h$ such that $h p$ is sos? Can we find a form $q$ so that $q^{2} p$ is sos? If we've answered the second, we've answered the first. Conversely, if $p \neq 0$ is psd and $h p$ is sos, then $h$ is psd. But it needn't be sos; indeed, a trivial answer to the first question is to take $h=p$. Stengle proved [25] that if $p(x, y, z)=x^{3} z^{3}+\left(y^{2} z-x^{3}-z^{2} x\right)^{2}$, then $p^{2 s+1} \in \Delta_{3,6(2 s+1)}$ for every integer $s$. That is, $p^{2 s-1} \cdot p$ is sos, but $p^{2 s} \cdot p$ is not. Choi and Lam showed [1] that for $S \in \Delta_{3,6}$ (see (3) below), the product $S(x, y, z) S(x, z, y)$ is actually sos.

The author gratefully acknowledges correspondence with Chip Delzell, Pablo Parrilo, Vicki Powers, Marie-Françoise Roy and Claus Scheiderer. Their suggestions have made this a better paper.

\section{WHAT IS KNOWN ABOUT THE DENOMINATOR}

The first concrete result about a denominator in Hilbert's 17th Problem was found by Pólya [17]. He showed that if $f \in H_{d}\left(\mathbb{R}^{n}\right)$ is positive on the unit simplex $\left\{\left(x_{1}, \ldots, x_{n}\right) \mid x_{j} \geq 0, \sum x_{j}=1\right\}$, then for sufficiently large $N,\left(\sum_{j} x_{j}\right)^{N} f$ has positive coefficients. Replacing each $x_{j}$ by $x_{j}^{2}$, we see that if $p \in H_{2 d}\left(\mathbb{R}^{n}\right)$ is an even positive definite form, then $\left(\sum_{j} x_{j}^{2}\right)^{N} p$ is a sum of even monomials with positive coefficients, and so, as it stands, is a sum of squares of monomials. Taking even $N$, we see that $q=\left(\sum_{j} x_{j}^{2}\right)^{N / 2}$ is a denominator for $p$. Habicht [7] generalized Pólya's proof to give an alternate solution to Hilbert's 17th Problem for pd forms; however, $h$ is not readily constructible and in general is no longer a power of $\sum x_{j}^{2}$. Except for one example, Pólya did not attempt to determine an explicit value of $N$. A good exposition of the theorems of Pólya and Habicht can be found in [8]. 
For positive definite $p \in P_{n, m}$, let

$$
\epsilon(p):=\frac{\inf \left\{p(u): u \in S^{n-1}\right\}}{\sup \left\{p(u): u \in S^{n-1}\right\}}
$$

measure how "close" $p$ is to having a zero. The author 19 showed that if

$$
N \geq \frac{n m(m-1)}{(4 \log 2) \epsilon(p)}-\frac{n+m}{2}
$$

then $\left(\sum_{j} x_{j}^{2}\right)^{N} p$ is a sum of $(m+2 N)$-th powers of linear forms, and so is sos. A similar lower bound has been shown to apply in Pólya's Theorem; the bound goes to infinity as $p$ approaches the boundary of $P_{n, m}$. (See papers by de Loera and Santos [13] and by Powers and the author [18.)

The restriction to positive definite forms is necessary. There exist psd forms $p$ in $n \geq 4$ variables so that, if $h^{2} p$ is sos, then $h$ must have a specified zero. The existence of these unavoidable singularities, or so-called "bad points", insures that $\left(\sum x_{j}^{2}\right)^{r} p$ can never be a sum of squares of forms for any $r$. Habicht's Theorem implies that no positive definite form can have a bad point. Bad points were first noted by Straus and have been extensively studied by Delzell; see, e.g. [5, 6].

Little specific is known about the degree of the denominator in more than 3 variables. A. Robinson proved [21 p. 268] that there exists $d(n, m)$ so that $p \in P_{n, m}$ implies that there exists $q \in H_{d(n, m)}\left(\mathbb{R}^{n}\right)$ so that $q^{2} p$ is sos. Moreover, $d(n, m)$ is a general recursive function of $n$ and $m$. Various improvements have been made in the description of $d$, but no "practical" bounds are known. See [4, $\S 5.4-5.6$, $5.11-5.13,9.1-9.7]$ for a detailed survey. The existence of $d(n, m)$ is also a special case of a quantitative version of the Positivstellensatz constructed by Lombardi and Roy [14.

\section{RECENT RESUlts AND A NEW THEOREM}

Scheiderer has shown in very recent work [24] that for $p \in P_{3, m}$, there exists $N=N(p)$ so that $\left(x^{2}+y^{2}+z^{2}\right)^{N} p(x, y, z)$ is sos; indeed, $x^{2}+y^{2}+z^{2}$ can be replaced by any positive definite form. This is a strong refutation to the existence of bad points for ternary forms.

Suppose $(n, m)$ is such that $\Delta_{n, m} \neq \emptyset$. Theorem 1 below states that there is no single form $h$ so that, if $p \in P_{n, m}$, then $h p$ is sos. Corollary 2 says that there is not even a finite set of forms $\mathcal{H}$ so that, if $p \in P_{n, m}$, then there exists $h \in \mathcal{H}$ so that $h p$ is sos. In particular, there does not exist a finite set of denominators which apply to all of $P_{n, m}$. This result implies that $N(p)$ in Scheiderer's theorem is not bounded as $p$ ranges over $P_{3, m}$. It also implies that the denominators in the Lombardi-Roy theorem cannot be chosen from a finite, predetermined set.

The proof of the theorem is elementary and relies on a few simple observations. If $p \neq 0$ is psd and $h p$ is sos, then $h$ is psd. As previously noted, $\Sigma_{n, m}$ is a closed cone for all $(n, m)$. This cone is invariant under the action of taking invertible linear changes of variable. Thus, if $h^{\prime}$ is derived from $h$ by such a linear change, and if $h p$ is sos for every $p \in P_{n, m}$, then so is $h^{\prime} p$. Suppose $\ell$ is a linear form, $p=\sum_{k} g_{k}^{2}$ is sos, and $\ell \mid p$. Then $\ell^{2} \mid p$ and $\ell \mid g_{k}$ for each $k$, and by induction, $\ell^{2 s}\left|p \Longrightarrow \ell^{s}\right| g_{k}$. Thus, we can "peel off" squares of linear factors from any sos form; this is a common practice, dating back at least to [22, p. 267]. We use this observation in the contrapositive: if $p \in \Delta_{n, m}$, then $\ell^{2 s} p \in \Delta_{n, m+2 s}$. 
Theorem 1. Suppose $\Delta_{n, m} \neq \emptyset$. Then there does not exist a non-zero form $h$ so that if $p \in P_{n, m}$, then $h p$ is sos.

Proof. Suppose to the contrary that such a form $h$ exists. Since $h \neq 0$, there exists a point $a \in \mathbb{R}^{n}$ so that $h(a) \neq 0$. By making an invertible linear change of variables, we can take $a=(1,0, \ldots, 0)$. Thus, we may assume without loss of generality that $h\left(x_{1}, 0, \ldots, 0\right)=\alpha x_{1}^{d}$, where $\alpha>0$ and $d$ is even. In the sequel, we distinguish $x_{1}$ from the other variables.

Choose $p \in P_{n, m} \backslash \Sigma_{n, m}$. Then

$$
h\left(x_{1}, x_{2}, \ldots, x_{n}\right) p\left(x_{1}, r x_{2}, \ldots, r x_{n}\right)
$$

is sos for every $r \in \mathbb{N}$. By making the change of variables $x_{i} \rightarrow x_{i} / r$ for $i \geq 2$, we see that

$$
h\left(x_{1}, r^{-1} x_{2}, \ldots, r^{-1} x_{n}\right) p\left(x_{1}, x_{2}, \ldots, x_{n}\right)
$$

is also sos. Since

$$
\lim _{r \rightarrow \infty} h\left(x_{1}, r^{-1} x_{2}, \ldots, r^{-1} x_{n}\right)=h\left(x_{1}, 0, \ldots, 0\right)=\alpha x_{1}^{d},
$$

and since $\Sigma_{n, m+d}$ is closed, it follows that

$$
\lim _{r \rightarrow \infty} h\left(x_{1}, r^{-1} x_{2}, \ldots, r^{-1} x_{n}\right) p\left(x_{1}, x_{2}, \ldots, x_{n}\right)=\alpha x_{1}^{d} p\left(x_{1}, \ldots, x_{n}\right)
$$

is sos. Thus $p$ is sos, a contradiction.

The following elegant proof is due to Claus Scheiderer and is included with his permission; it supersedes the proof in an earlier version of this manuscript.

Corollary 2. Suppose $\Delta_{n, m} \neq \emptyset$. Then there does not exist a finite set of non-zero forms $\mathcal{H}=\left\{h_{1}, \ldots, h_{N}\right\}$ with the property that, if $p \in P_{n, m}$, then $h_{k} p$ is sos for some $h_{k} \in \mathcal{H}$.

Proof. Suppose $\mathcal{H}$ exists. For each $k$, there exists non-zero $p \in \Delta_{n, m}$ so that $h_{k} p$ is sos. (Otherwise, we may delete $h_{k}$ harmlessly from $\mathcal{H}$.) Thus, each $h_{k}$ is psd, and there exists a form $q_{k}$ so that $q_{k}^{2} h_{k}$ is sos. Define $h=\prod_{k} q_{k}^{2} h_{k}$. We now show that for every $p \in P_{n, m}, h p$ is sos: this contradicts Theorem 1 and proves the corollary. By hypothesis, there exists $h_{j} \in \mathcal{H}$ so that $h_{j} p$ is sos. Thus,

$$
h p=\left(\prod_{k \neq j} q_{k}^{2} h_{k}\right) \cdot q_{j}^{2} \cdot h_{j} p
$$

is a product of sos factors, and so is sos.

Finally, we know by Hilbert's theorem that for $p \in P_{3,6}$, there exists quadratic $h$ so that $h p \in \Sigma_{3,8}$. The three simplest forms in $\Delta_{3,6}$ are

$$
M(x, y, z)=x^{4} y^{2}+x^{2} y^{4}+z^{6}-3 x^{2} y^{2} z^{2}, \quad \text { due to Motzkin [15]; }
$$

R. M. Robinson's 22] simplification of Hilbert's construction

(2) $R(x, y, z)=x^{6}+y^{6}+z^{6}-\left(x^{4} y^{2}+x^{2} y^{4}+x^{4} z^{2}+x^{2} z^{4}+y^{4} z^{2}+y^{2} z^{4}\right)+3 x^{2} y^{2} z^{2}$;

and

$$
S(x, y, z)=x^{4} y^{2}+y^{4} z^{2}+z^{4} x^{2}-3 x^{2} y^{2} z^{2}, \quad \text { due to Choi and Lam [1, 2]. }
$$


It is not too difficult to consider $q M, q R, q S$ for $q(x, y, z)=a^{2} x^{2}+b^{2} y^{2}+c^{2} z^{2}$ and determine whether these are sos using the algorithm of [3] directly or its implementation in, e.g., [16].

Interestingly enough, these conditions are the same in each case: the forms are sos if and only if

$$
2\left(a^{2} b^{2}+a^{2} c^{2}+b^{2} c^{2}\right) \geq a^{4}+b^{4}+c^{4} .
$$

This expression factors rather neatly into

$$
(a+b+c)(a+b-c)(b+c-a)(c+a-b) \geq 0,
$$

so if $a \geq b \geq c \geq 0$ without loss of generality, the only non-trivial condition is that $b+c \geq a$; that is, there is a (possibly degenerate) triangle with sides $a, b, c$. (Robinson [22, p. 273] has a superficially similar condition, but note that his multiplier is $a x^{2}+b y^{2}+c z^{2}$.)

If we scale variables as in the proof of Theorem 1 , it follows from this computation that the three forms

$\left(x^{2}+y^{2}+z^{2}\right) M(x, \lambda y, \lambda z),\left(x^{2}+y^{2}+z^{2}\right) R(x, \lambda y, \lambda z),\left(x^{2}+y^{2}+z^{2}\right) S(x, \lambda y, \lambda z)$

are sos if and only if $0 \leq|\lambda| \leq 2$.

\section{REFERENCES}

[1] Choi, M. D. and T. Y. Lam, An old question of Hilbert, Queen's Papers in Pure and Appl. Math. (Proceedings of Quadratic Forms Conference, Queen's University (G. Orzech ed.)), 46 (1976), 385-405. MR0498375 (58:16503)

[2] Choi, M. D. and T. Y. Lam, Extremal positive semidefinite forms, Math. Ann., 231 (1977), 1-18. MR 0498384 (58:16512)

[3] Choi, M. D., T. Y. Lam and B. Reznick, Sums of squares of real polynomials, Proc. Sympos. Pure Math., 58.2 (1995), 103-126. MR1327293 (96f:11058)

[4] Delzell, C. N., Kreisel's unwinding of Artin's proof in Kreiseliana about and around Georg Kreisel (P. Odifreddi ed.), A. K. Peters, Wellesley, 1996, 113-246. MR.1435764

[5] Delzell, C. N., Bad points for positive semidefinite polynomials, Abstracts Amer. Math. Soc., 18 (1997), \#926-12-174, 482.

[6] Delzell, C. N., Unavoidable singularities when writing polynomials as sums of squares of real rational functions, in preparation.

[7] Habicht, W., Über die Zerlegung strikte definiter Formen in Quadrate, Comment. Math. Helv., 12 (1940) 317-322. MR0002837 (2:119f)

[8] Hardy, G. H., J. E.. Littlewood and G. Pólya, Inequalities, Cambridge Univ. Press, 2nd ed., 1967. MR0944909 (89d:26016)

[9] Hilbert, D., Uber die Darstellung definiter Formen als Summe von Formenquadraten, Math. Ann. 32 (1888), 342-350; see Ges. Abh. 2, 154-161, Springer, Berlin, 1933, reprinted by Chelsea, New York, 1981.

[10] Hilbert, D., Über ternäre definite Formen, Acta Math. 17 (1893), 169-197; see Ges. Abh. 2, 345-366, Springer, Berlin, 1933, reprinted by Chelsea, New York, 1981.

[11] de Klerk, E. and D. V. Pasechnik, Products of positive forms, linear matrix inequalities, and Hilbert 17-th problem for ternary forms, European J. of Oper. Res. 157 (2004), 39-45. MR 2064275

[12] Landau, E., Über die Darstellung definiter Funktionen durch Quadrate, Math. Ann., 62 (1906), pp. 272-285; also in Collected Works, vol. 2, pp. 237-250, Thales-Verlag, Essen, 1986. MR:0937897 (92b:01082b)

[13] de Loera, J. A. and F. Santos, An effective version of Pólya's theorem on positive definite forms, J. Pure Appl. Algebra, 108 (1996), 231-240. (See correction, same journal, 155 (2001), 309-310.) MR1384003 (97b:12001) MR:1801421 (2001m:11058)

[14] Lombardi, H. and M.-F. Roy, Elementary recursive degree bounds for Positivstellensatz, in preparation. 
[15] Motzkin, T, S., The arithmetic-geometric inequality, pp. 205-224 in Inequalities (O. Shisha, ed.) Proc. of Sympos. at Wright-Patterson AFB, August 19-27, 1965, Academic Press, New York, 1967; also in Theodore S. Motzkin: Selected Papers, Birkhäuser, Boston, 1983 (D. Cantor, B. Gordon and B. Rothschild, eds.). MR0223521 (36:6569)

[16] Parrilo, P., Structured semidefinite programs and semialgebraic methods in robustness and optimization, Ph.D. thesis, Calif. Inst. of Tech., 2000.

[17] Pólya, G., Über positive Darstellung von Polynomen, Vierteljschr. Naturforsch. Ges. Zürich, 73 (1928), 141-145; see Collected Papers, Vol. 2, pp. 309-313, MIT Press, Cambridge, Mass., London, 1974. MR.0505094 (58:21342)

[18] Powers, V. and B. Reznick, A new bound for Pólya's theorem with applications to polynomials positive on polyhedra, J. Pure Appl. Algebra 164 (2001), 221-229. MR.1854339 (2002g:14087)

[19] Reznick, B., Uniform denominators in Hilbert's Seventeenth Problem, Math. Z., 220 (1995), 75-98. MR,1347159 (96e:11056)

[20] Reznick, B., Some concrete aspects of Hilbert's 17th Problem, Contemp. Math., 253 (2000), 251-272. MR1747589 (2001i:11042)

[21] Robinson, A., On ordered fields and definite forms, Math. Ann., 130 (1955), 257-271. MR0075932(17:822a)

[22] Robinson, R. M., Some definite polynomials which are not sums of squares of real polynomials, Izdat. "Nauka" Sibirsk. Otdel. Novosibirsk, (1973) pp. 264-282, (Selected questions of algebra and logic (a collection dedicated to the memory of A. I. Mal'cev), abstract in Notices AMS, 16 (1969), p. 554. MR0337878 (49:2647)

[23] Rudin, W., Sums of squares of polynomials, Amer. Math. Monthly, 107 (2000), 813-821. MR $1792413(2002 \mathrm{c}: 12003)$

[24] Scheiderer, C., Sums of squares on real algebraic surfaces, preprint.

[25] Stengle, G., Integral solution of Hilbert's seventeenth problem, Math. Ann. 246 (1979/1980), 33-39. MR.0554130 (81c:12035)

[26] Swan, R.G., Hilbert's theorem on positive ternary quartics, Contemp. Math. 272 (2000), 287-292. MR1803372 (2001k:11065)

Department of Mathematics, University of Illinois at Urbana-Champaign, Urbana, ILLINOIS 61801

E-mail address: reznick@math.uiuc.edu 